\title{
Automated Detection of Rapid Variability of Moss Using SDO/AIA and Its Connection to the Solar Corona
}

\author{
David R. Graham ${ }^{1}$, Bart De Pontieu ${ }^{2,3,4}$ (1), and Paola Testa ${ }^{5}$ (1) \\ ${ }^{1}$ Bay Area Environmental Research Institute, Moffett Field, CA 94035, USA; graham@1msal.com \\ ${ }^{2}$ Lockheed Martin Solar \& Astrophysics Lab, Org. A021S, Bldg. 252, 3251 Hanover Street, Palo Alto, CA 94304, USA \\ ${ }^{3}$ Rosseland Centre for Solar Physics, University of Oslo, P.O. Box 1029 Blindern, NO-0315, Oslo, Norway \\ ${ }^{4}$ Institute of Theoretical Astrophysics, University of Oslo, P.O. Box 1029 Blindern, NO-0315, Oslo, Norway \\ ${ }^{5}$ Harvard - Smithsonian Centre for Astrophysics, 60 Garden St., Cambridge, MA 02138, USA \\ Received 2019 May 30; revised 2019 July 2; accepted 2019 July 3; published 2019 July 19
}

\begin{abstract}
Active region moss - the upper transition region of hot loops-was observed exhibiting rapid intensity variability on timescales of order $15 \mathrm{~s}$ by Testa et al. in a short time series $(\sim 150 \mathrm{~s})$ data set from Hi-C (High-resolution Coronal Imager). The intensity fluctuations in the subarcsecond 193A images $(\sim 1.5$ MK plasma) were uncharacteristic of steadily heated moss and were considered an indication of heating events connected to the corona. Intriguingly, these brightenings displayed a connection to the ends of transient hot loops seen in the corona. Following the same active region, AR11520, for 6 days, we demonstrate an algorithm designed to detect the same temporal variability in lower resolution Atmospheric Imaging Assembly (AIA) data, significantly expanding the number of events detected. Multiple analogous regions to the Hi-C data are successfully detected, showing moss that appears to "sparkle" prior to clear brightening of connected high-temperature loops; this is confirmed by the hot AIA channels and the isolated Fe XVIII emission. The result is illuminating, as the same behavior has recently been shown by Polito et al. while simulating nanoflares with a beam of electrons depositing their energy in the lower atmosphere. Furthermore, the variability is localized mostly to the hot core of the region, hence we reinforce the diagnostic potential of moss variability as the driver of energy release in the corona. The ubiquitous nature of this phenomenon, and the ability to detect it in data with extended time series, and large fields of view, opens a new window into investigating the coronal heating mechanism.
\end{abstract}

Key words: Sun: activity - Sun: chromosphere - Sun: flares - Sun: transition region - Sun: UV radiation - Sun: $\mathrm{X}$-rays, gamma rays

\section{Introduction}

Heating of the corona, the outer atmosphere of the Sun, is predicted to occur via several modes of energy release. One proposal is the constant dissipation of magnetohydrodynamic waves into the surrounding plasma, while another is through impulsive events at frequencies high enough to overcome the natural plasma cooling time. It is thought that, similar to larger flares ,there is a distribution of increasingly small and more frequent events (Hannah et al. 2011), and that they too originate from a reconnecting magnetic field. The released energy is redistributed into the plasma either via particle acceleration and collisions (Brown 1971), waves (Fletcher \& Hudson 2008), or thermal conduction. These "nanoflares" in principle should have hard X-ray spectra analogous to those of large flares-with a non-thermal tail in the photon spectrum. However, up until very recently (Wright et al. 2017) such signatures have been below detection limits, and even now they are only detectable at low spatial resolution. Fortunately, highresolution imaging or spectroscopy in the ultraviolet or extreme-ultraviolet (EUV) can help us probe the energy release indirectly, through changes in the intensity of temperaturesensitive emission lines (see Testa et al. 2013, 2014; Viall \& Klimchuk 2016). Recent modeling (Testa et al. 2014; Polito et al. 2018) has placed further constraints on the observables expected from different heating mechanisms, and the parameters of the non-thermal particle distribution.

Moss, named after its clumpy appearance in images, is the result of $1 \mathrm{MK}$ plasma occurring in the transition region (TR) of hot and high pressure loops (e.g., Fletcher \& de
Pontieu 1999). Bright in the diagnostic-rich EUV, moss is a valuable window into the interface between the chromosphere and corona. Until recently, moss was seen as a relatively stable phenomenon, showing low-intensity oscillations over timescales of hours (Antiochos et al. 2003), with some shorter oscillations, of the order of minutes, driven by chromospheric motions (De Pontieu et al. 2003). More pertinent to nanoflare studies are recent observations of higher-frequency intensity modulation. Testa et al. (2013) found strong variability in highresolution $193 \AA$ imaging of moss from the Hi-C rocket flight, where it showed a "twinkling" appearance in movies. The bright spots were small $\left(<1^{\prime \prime}\right)$ and confined to the feet of very hot $(T>5 \mathrm{MK})$ coronal loops, their connection determined from $94 \AA$ Atmospheric Imaging Assembly (AIA) images. The authors concluded that the rapid moss variability (RMV), on the order of 15-30 s was a signature of coronal nanoflare heating. Testa et al. (2014) later showed the same variability in the IRIS Si IV TR line.

Similar events have been observed showing IRIS TR brightenings at the footpoints of transient hot loops (P. Testa et al. 2019, in preparation). These studies, while enlightening, were based on manually selected small field-of-view (FOV) data that do not build statistically significant samples to provide clues about the frequency and relevance of these events to active region (AR) heating. Our motivation is to investigate the distribution of these events in long-duration, large FOV data sets from the AIA (Lemen et al. 2012) on board the Solar Dynamics Observatory (SDO; Pesnell et al. 2012). The relation of RMV to the magnetic and plasma environment will help 
determine the source of rapid heating, the magnetic conditions leading to RMV, and crucially, if it is a significant driver of heating over the lifetime of an AR.

We show in this Letter that the $\sim 12 \mathrm{~s}$ cadence AIA imaging at $193 \AA$ and $171 \AA$ is sensitive to intensity fluctuations of $<30$ s, demonstrating that the now $10 \mathrm{yr}$ of $S D O$ data holds a wealth of information on moss nanoflare events. We introduce an algorithm to detect these events and test it on a 6 day data set. Section 2 describes the data preparation; Section 3 describes the detection algorithm. Section 4 shows the distribution of RMV plus examples of loop heating, and Section 5 discusses the influence of the magnetic and plasma environment on RMV.

\section{Data and Analysis}

We follow the evolution of AR11520 for 6 days during its passage across the solar disk from 2012-07-9T00:00 to 201207-15T00:00, overlapping and bracketing the Hi-C flight that took data from 2012-07-11T18:52:49 to 2012-07-11T18:56:10. We take data from AIA, at $12 \mathrm{~s}$ cadence, and the Helioseismic and Magnetic Imager (HMI; Scherrer et al. 2012) at $45 \mathrm{~s}$ cadence; it is also on board SDO. The data were prepared with the AIA_PREP and HMI_PREP routines. To highlight hightemperature loops we isolate the Fe XVIII $93.932 \AA$ line emission ( 7 MK) from the AIA $94 \AA$ channel by subtracting an estimate of the contribution from cooler lines of the same ion species within the $171 \AA$ and $211 \AA$ filters, where the estimates are given by the formula in Del Zanna 2013.

Figure 1 captures the region at three times in AIA $171 \AA$, Fe XVIII, and AIA $193 \AA$ (top, second, and third rows), and the line-of-sight magnetic flux from HMI (fourth row). In the left panels, the start of our data set, the region is well established at its appearance on the limb, with two major positive and negative polarity spots. A smaller, concentrated area of negative flux (black) is found at the bottom of a fan loop $\left(-710^{\prime \prime},-220^{\prime \prime}\right)$, clear in $171 \AA$. Emerging flux is present in the southwest $\left(-510^{\prime \prime},-380^{\prime \prime}\right)$ and accompanied by complex loops, becoming host to numerous small flares and nanoflare detections (Section 4). The dark filament in the middle of the region (center frames) is a source of small explosive events.

\section{Detection Method}

\subsection{Location}

Our aim is to isolate moss intensity fluctuations shorter than $30 \mathrm{~s}$ with connections to overlying hot loops. The following procedure uses a series of thresholds in different AIA filters to isolate areas of "twinkling" moss. We first locate bright chromospheric emission in AIA $1700 \AA$ images using a constant intensity threshold (Figure 2(a)). The loop regions in Figure 2(b) are identified by taking the isolated Fe XVIII line at $7 \mathrm{MK}$ and defining a contour encompassing continuous loop structures. We restrict the minimum loop area to 100 pixels, as we expect loops with footpoint connections to have appreciable length. Another threshold in $193 \AA$ (Figure 2(c)) includes only the bright moss emission. All of the selected regions are enlarged slightly to include small regions and ensure some overlap between filters. The combination of loop, moss, and network filters is shown by the green contours in Figure 2(d) over the HMI magnetogram. These selected areas are used to mask the running difference detection described below.
Large flares and filament eruptions also create rapid rises in intensity, therefore we flag these as false positives as here we focus on the heating mechanisms of quiescent AR loops. To remove these we obtain the differential emission measure (DEM) using the code by Cheung et al. (2015), performing an inversion on the $94,131,171,193,211$, and $335 \AA$ filters, as we expect the emission measure of these large events to be greater than that in nanoflare events. Using the DEM we eliminate events in two-temperature bands, between $\log T=5.6-5.8$ and $\log T=6.7-7.0$, with emission measures greater than $2 \times 10^{26}$ and $2 \times 10^{27} \mathrm{~cm}^{-5}$, respectively. We also perform the DEM over data binned at four times the nominal AIA cadence, spreading (in space) dynamic, growing features, e.g., post-flare arcades. Removing these prevents spikes in the detection count that are unrelated to the twinkling moss. An example selection is seen in Figure 2(e) and is overlaid in blue on Figure 2(b). There, the DEM filter removes the end of a bright flaring loop in the south, along with several smaller, elongated brightenings.

\subsection{Temporal Selection}

Running 1-step difference images for both $171 \AA$ and $193 \AA$ are used to detect pixels with rapid intensity variations. First, a cross-correlation is performed in time and between wavelengths to remove any pointing jitter. The total measured uncertainty $(\sigma)$ for both filters is calculated from the AIA_BP_ESTIMATE_ERROR routine. We then use a modified version of the method from Testa et al. (2013) to search the running difference for zero-crossings (changes in sign) above a threshold of $4 \sigma$, indicating rapid brightness change. Pixels identified in both $171 \AA$ and $193 \AA$ are selected. The $171 \AA$ selection is enlarged by 1 pixel in all directions to account for residual pointing differences. The detected events for the example time step, after applying the above filters, are shown in red in Figure 2(f) over the $193 \mathrm{~A}$ difference image.

\section{Results}

\subsection{By Example}

For validation of our results. in Figure 3 (top panel) we show the light curves and detected events from 4 AIA pixels (black) within the Hi-C area studied by Testa et al. (2013). As Hi-C oversamples AIA $(\sim 0$ ". 1 pixel size to $\sim 0$ ". 6$)$ we plot above each AIA light curve the closest matching Hi-C curve (gray) within the AIA pixel boundaries. Like Testa et al. (2013), our algorithm detects AIA pixels with significant zero-crossings following the criteria in Section 3. Detections are marked by blue diamonds on the original AIA light curve in Figure 3. Since the zero-crossing can be positive or negative, the detection may fall on, or immediately after the intensity change. The largest sub-minute changes in AIA are detected successfully, e.g., at 18:52 UT in the second curve from the bottom, and where rapid changes are also seen in $\mathrm{Hi}-\mathrm{C}$, e.g., the bottom curve at 18:55 UT. Hi-C does reveal finer intensity variability, however, our AIA algorithm avoids confusion with longer-period variability well, showing that AIA is more than capable of identifying sub-minute heating and cooling signatures. The AIA data shows how fortunate $\mathrm{Hi}-\mathrm{C}$ was to detect such signatures of RMV, as prior to the Hi-C data set (18:53 UT), the area showed little variability.

The intensity thresholds selecting the moss, network, and loop areas in Figure 2 are quite robust, i.e., the quiescent moss 


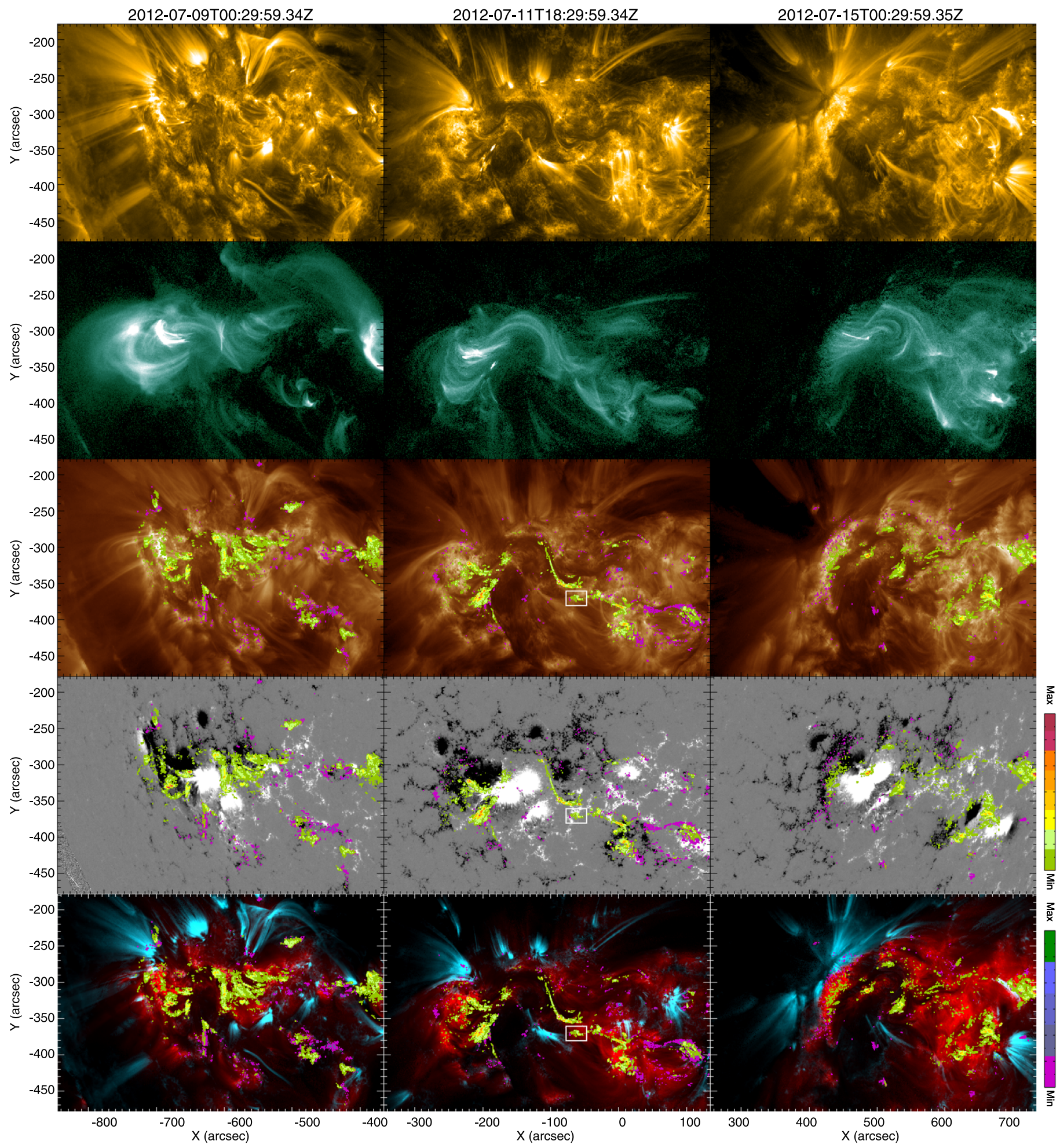

Figure 1. Context images for the active region at the east limb, on disk, and at the west limb. From top to bottom: AIA $171 \AA$; the isolated Fe XVIII extracted from AIA $94 \AA$ images; AIA $193 \AA$ A HMI magnetic flux (white as positive); and the emission measure at 800,000 K (blue) and $2.5 \mathrm{MK}$ (red). The total variable moss detections within one hour are overplotted in rows three through five using the upper color table for events within the Fe XVIII loop restriction, and the lower color table for events outside it (both are scaled with the detection number). The hour is centered on the AIA $171 \AA$ timestamp. The solid white box marks a region of variability in the Hi-C data (Testa et al. 2013).

and loop intensities do not change appreciably throughout the entire data set, and are much lower, and relatively constant, compared to the RMV and flares. However, the zero-crossing threshold above the measurement uncertainty can significantly increase the detected event number if set too low. We use the events observed with Hi-C to calibrate the choice of sigma and determine what is an acceptable level of noise versus RMV detection. At $4 \sigma$, the Hi-C RMV patches are detectable in AIA. In lowering the threshold to $3 \sigma$ the mean event number per frame between 2012-07-11T18:00:00-19:00:00 increases from 


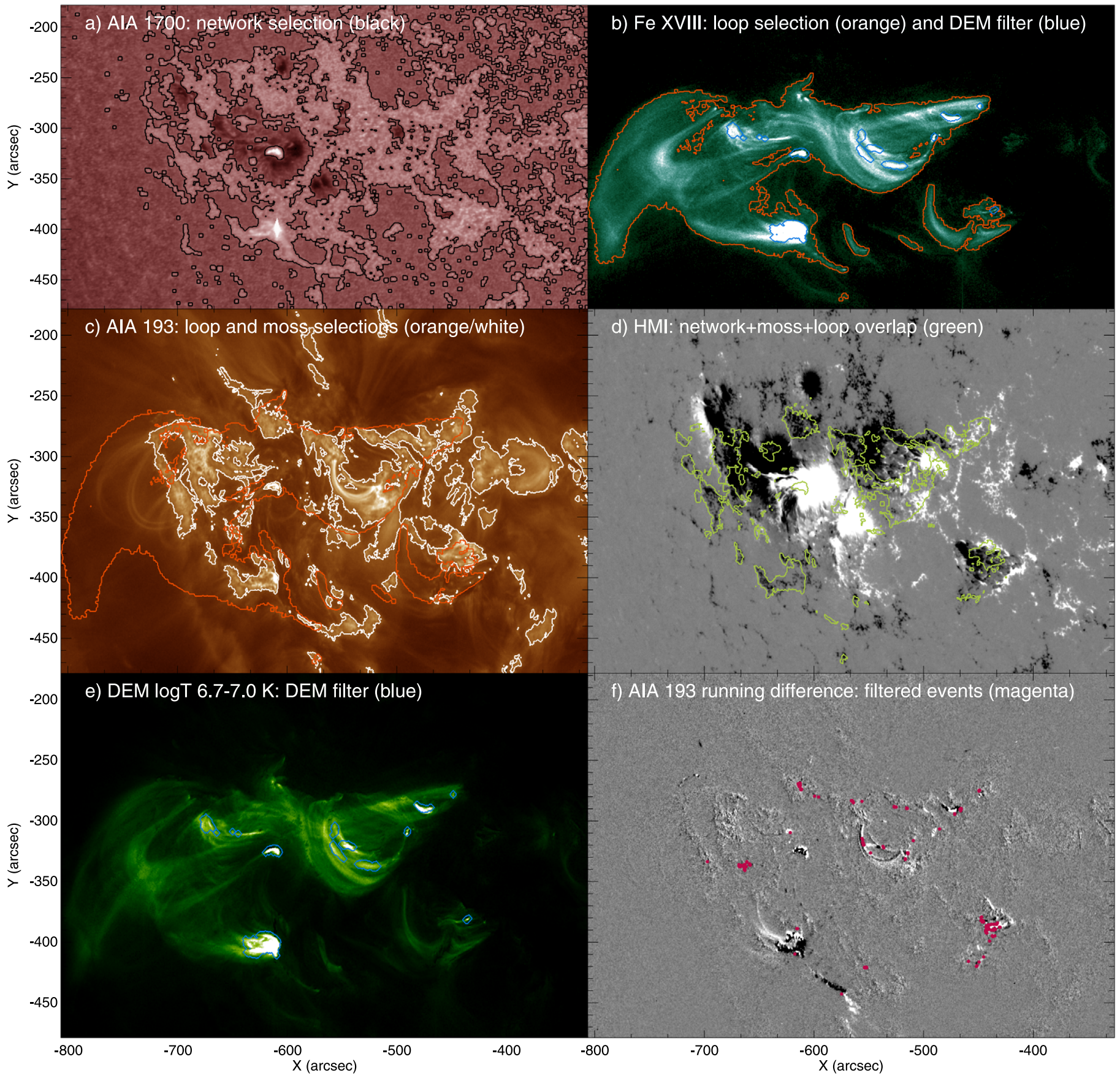

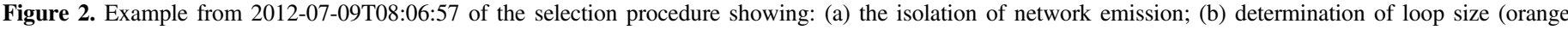

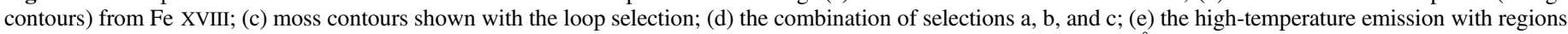
to be excluded; (f) final selected events with rapid moss variability (red) shown on the running difference of the $193 \AA$ intensity.

78 to 125 . At $2 \sigma$, this jumps to $\sim 500$ and the spatial distribution of detections becomes noisy. Thus, our choice of $4 \sigma$ may err on the conservative side, but the detection limit of AIA seems to be close to $3 \sigma$.

In Figure 4 we look at three different loop systems in Fe XVIII with strong moss variability. In the first example, the first six exposures show an increase of moss detections (pink) while growing Fe XVIII brightness reveals heating loops connecting the moss sources. By the final exposure the moss activity has almost ceased and the loop remains bright. The corresponding HMI magnetogram shows a typical scenario with footpoints forming at opposing polarities - this final frame shows the cumulative events over the exposures displayed.

The next example is located in the trailing half of the AR close to the polarity inversion line. Scattered events are identified before forming a brightening ribbon (bright even in Fe XVIII) in the center left of the images. Its opposite polarity counterpart (see final HMI frame) forms a little later, from Exposure 13 on. After $\sim 8$ minutes the loop system connecting the two ribbon areas has become bright and the moss events have subdued.

The final example is from the same area some $18 \mathrm{hr}$ later. As before, moss events precede enhancements of hotter material 

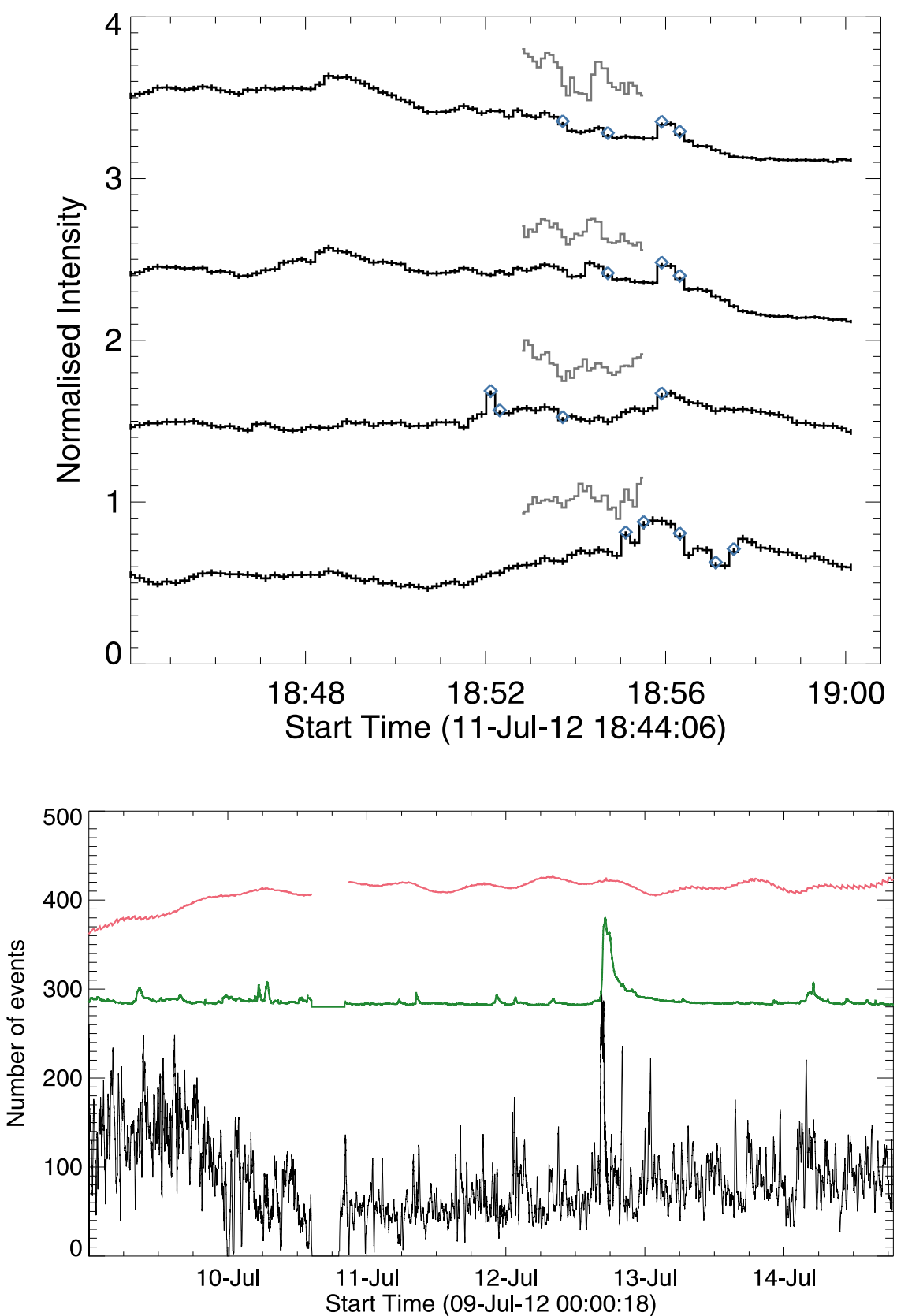

Figure 3. Top: AIA light curves (black) for four example pixels with detections from the region observed by Hi-C (Testa et al. 2013) (white box in Figure 1). The blue diamonds mark a detection, a zero-crossing in the running difference above $4 \sigma$. Normalized Hi-C light curves ( 0 "! 1 pixel) from the corresponding co-aligned AIA pixel $(0$. " 6 ) are shown in gray above each AIA light curve. Bottom: total number of detected events in the FOV (black), the total normalized Fe XVIII intensity (green), and the unsigned flux determined from HMI normalized and corrected for viewing angle (pink).

even though it is now a more complex system with more activations occurring along a set of sheared loops. The shearing is clear in Exposures 7 and 27, where the corresponding opposite footpoints now have a much larger north-south offset.

While the examples of Figure 4 show RMV with characteristics similar to a flare ribbon, tracing out an elongated structure, we note that in many of the events, like the Hi-C region from Testa et al. (2013), the RMV is more isolated and point-like, although still connected to high-temperature loops. The mix of RMV scales is evident in the bottom right panel of Figure 2.

\subsection{Global Picture}

Throughout the 6 day time series, we find that RMV often precedes intensifying hot loops visible in Fe XVIII. In the lower three rows of Figure 1 we show the total number of events in three one hour segments. In light green to red (upper color table) are the events located by the algorithm using all of the criteria outlined in Figure 2. To compare with the activity in cooler areas we show the events excluded by the hot loop restriction in magenta to dark green (lower color table). The color tables are scaled with increasing event frequency.

Typically, events are found in the bright core of the region, and near the new emergence in the bottom right of the FOV. Some areas harbor more repeated events, e.g in the complex 

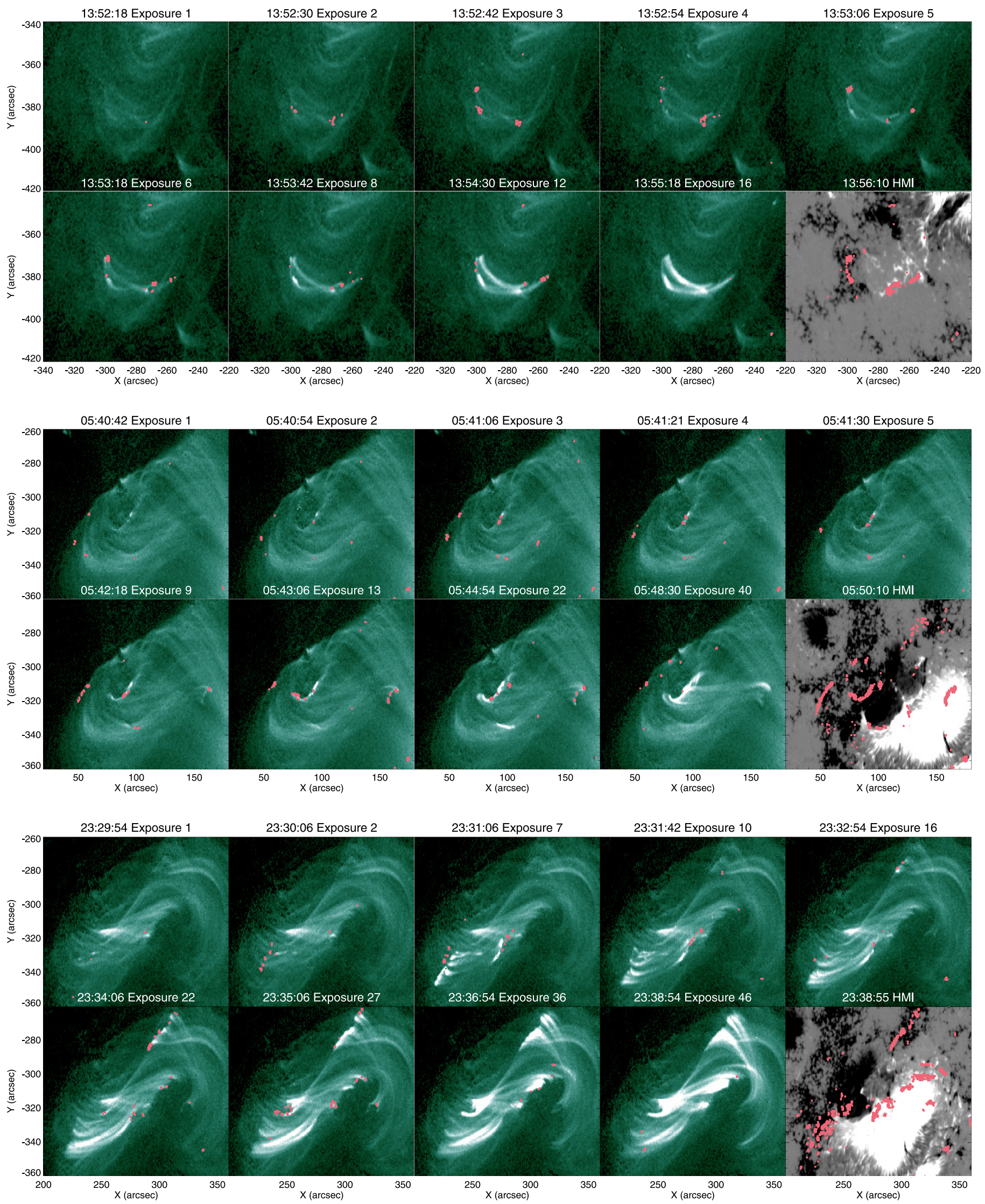

Figure 4. Three isolated heating events shown with Fe XVIII images from 2012 July 11 (top) and 2012 July 13 (middle and bottom panels). Selected exposures show the initial moss activity (detections in pink), followed by growing Fe XVIII loop emission ( $\sim 7 \mathrm{MK})$. The final HMI magnetogram is overplotted with the cumulative detections between the first and last exposures. 


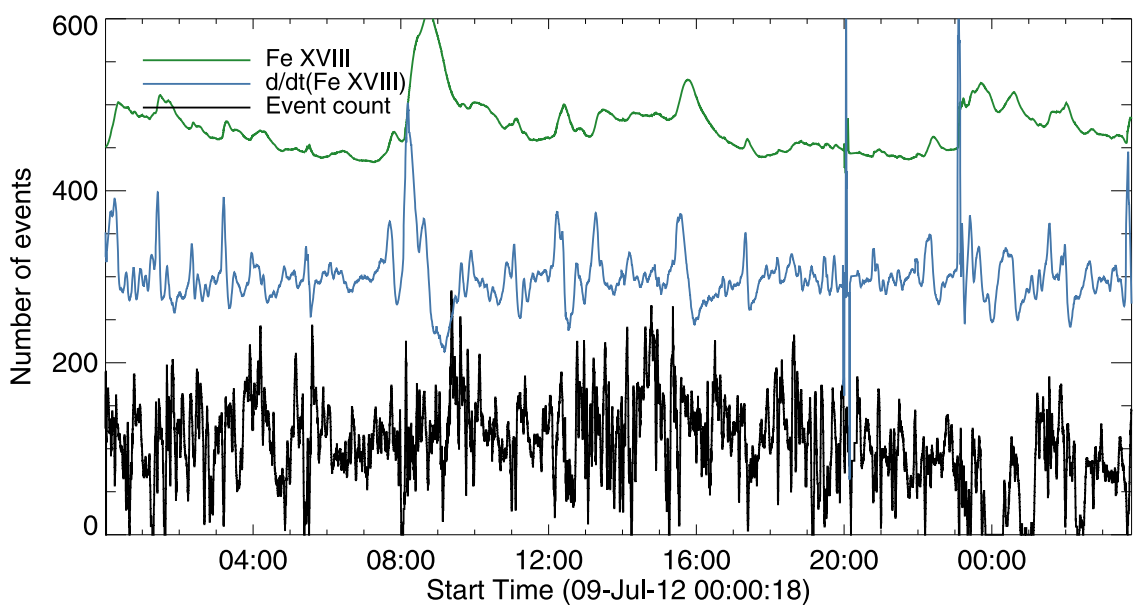

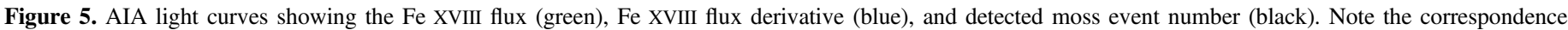
between the derivative and event number in the larger events.

area to the left half of the first column, while others, like those in the Hi-C observation (white box), occur only for a few minutes (see Figure 3).

More events are revealed outside of the loop restriction in the newly emerging region. Small patches are also found at the feet of the fan loops, although they are relatively infrequent. In the final row of Figure 1 we show a two-temperature image from the obtained DEM, $\log T=5.9$ in blue and $\log T=6.4$ in red. In general, events within the area defined by the hot AR core are far more common than those outside.

We show the time derivative of the spatially integrated Fe XVIII emission with the moss event number and integrated Fe XVIII flux in Figure 5. Here, the largest flux enhancements have good correspondence between the derivative and the event number, followed by an increase of overall Fe XVIII flux. This is qualitatively similar to the Neupert effect (Neupert 1968) seen in larger flares, where a correlation is found between the time derivative of soft X-ray flux and the more impulsive hard $\mathrm{X}$-ray flux. This observation suggests that the high-temperature emission could mostly be associated with transient events.

\section{Discussion and Conclusions}

From our analysis we demonstrate that the RMV observed in the Hi-C FOV Testa et al. (2013) is ubiquitous throughout the $\mathrm{AR}$ and over several days of observation. RMV is apparent immediately before visible heating of hot, transient Fe XVIII loops. We therefore propose that it is a clear signature of coronal heating driven by nanoflares.

The temporal behavior of RMV shows similarities with the standard flare model such as compact footpoints that energize quickly and are connected by loops filling with evaporated material (Falchi et al. 1997; Krucker et al. 2011). Numerical simulations for flares are available with flexible modes of energy release, e.g., using a single electron beam input (Uitenbroek 2001; Allred et al. 2015), or a multi-threaded approach (Reep et al. 2018). The RMV events can be modeled using assumptions of non-thermal electron beam fluxes constrained from nanoflare observations (Testa et al. 2014). Polito et al. (2018) present simulations of nanoflare-size rapid heating events $\left(6 \times 10^{24} \mathrm{erg}\right.$ total energy) using a radiativehydrodynamic simulation (see Carlsson \& Stein 1997; Allred et al. 2015, RADYN)). The authors show that energy release on short timescales through electron beams at the apex of a $1 \mathrm{MK}$ loop yields a dynamic, short-lived response in the underlying TR, following the thermalization of the non-thermal electrons in the dense plasma, similar to what we observe in our data. In loops with higher pre-nanoflare densities, the electron beams thermalize higher in the loops so that the impulsive TR behavior is less pronounced, i.e., gradual heating and slower flows. In this scenario, reconnection continuing after the initial loops have been filled with evaporating plasma (Figure 4) would result in continued heating, although the bursts detected by our algorithm will subside, as the majority of heating retreats from the footpoints into the loop itself. The effect is compatible with Figure 4 as the loop reaches maximum brightness and the relative intensity rise decreases. The result, however, cannot directly determine if the local field has exhausted its immediate potential for reconnection and heating. In either scenario, our detection algorithm would thus be a diagnostic of nanoflare heating associated with electron beams in newly forming or low-density loops; in other words, it is a lower limit to the total number of such events in the corona.

One very interesting result from our analysis is the apparent difference in RMV event frequency between the core of the region and the footpoints of cool fan loops, which show fewer events (see Figure 1, bottom row). The underlying reason for this difference could inform us of the nature of the energy release in these two distinct regions.

Several factors can impact the visibility of RMV: the density along the loops, the size and duration of nanoflares, and whether non-thermal electrons are generated in the nanoflare. Detailed numerical modeling will be required to settle this important issue, but our data allow us to speculate about several of these issues.

If we assume that nanoflares with similar size and duration to those found in the AR core were generated in the fan loops, there are implications for where these electron beams are generated. This is because the models described above suggest that the loop column mass plays a key role in the appearance of RMV. This modeling result is compatible with our finding that RMV appears just before new hot core loops. Figure 4 suggests that in the core, a mix of newly forming (initially low density) and mature (high density, post-evaporation) loops exist. Perhaps the footpoint variability is less common in cool fan loops because they have a larger column mass than hot core loops and/or do not show the same mix of newly forming empty loops and mature loops? The densities in cool fan loops 
are typically lower $\left(\log n_{e}=8.7-9.3\right.$ Tripathi et al. 2008a, 2008b) than those in the moss (typically $\log n_{e}=9.5$ ), so if a larger column mass were the cause for the different visibility of RMV, it would imply that the beams in cool fan loops would have to be generated much higher in the corona.

Another possibility is that the conditions governing magnetic energy release are different in fan loops compared to core loops. Perhaps in fan loops, reconnection and resulting nanoflare events are smaller and/or of longer duration, or non-thermal electrons are not generated as often, or with lower energy? Such differences would be compatible with the observational finding that the AR core is populated mostly by compact, lower-lying loops, with regular transient brightenings in Fe XVIII (shown in Ugarte-Urra \& Warren 2014), which differ from the longer, more stable loops toward the edges of the region. The field in the core may form more complex geometries, where the interaction of existing spots, or emerging flux, creates areas of non-parallel field forming significant angles between adjacent loops. A recent study by Reale et al. (2019) demonstrates that large angle reconnection is common in a set of nanoflare observations displaying hot Fe XVIII loops, like those of Figure 4. Figure 3 clearly demonstrates that some areas are not always active and as discussed in Testa et al. (2013), await the onset of slipping reconnection.

Further indication of the role of the magnetic environment is seen in Figure 3, where on the first day there is a slow rise in total unsigned magnetic flux (pink) and detected event frequency (black). The density of events in the area certainly points to a link between emerging regions and RMV; however, it is not yet clear how emergence leads to the reconnection presumably required to accelerate electron beams (and cause RMV) without further studies. In conclusion, the RMV is an excellent diagnostic of the underlying energy release mechanism and the conditions leading to coronal heating. A wider survey of ARs should reveal if the RMV is always associated with "flare-like" loop activations, if RMV events in the core are indeed more common, and if they are influenced by the AR age. In particular, the algorithm can now be applied to the prepared, co-aligned IRIS and AIA data cubes that are now available (see IRIS Technical Note 32), whereby one can search for events directly under the spectrograph slit for more detailed analyses.

This work is supported by NASA under contract NNG09FA40C (IRIS). P.T. acknowledges support by NASA grants NNX15AF50G and NNX15AF47G, and by contracts 8100002705 and SP02H1701R from Lockheed-Martin to SAO. This letter has benefited from discussions at a meeting of team "New diagnostics of particle acceleration in solar coronal nanoflares from chromospheric observations and modeling" at the International Space Science Institute (ISSI) in Switzerland. IRIS is a NASA small explorer mission developed and operated by LMSAL with mission operations executed at the NASA Ames Research center and major contributions to downlink communications funded by ESA and the Norwegian Space Centre.

\section{ORCID iDs}

Bart De Pontieu (D) https://orcid.org/0000-0002-8370-952X

Paola Testa (ii) https://orcid.org/0000-0002-0405-0668

\section{References}

Allred, J. C., Kowalski, A. F., \& Carlsson, M. 2015, ApJ, 809, 104 Antiochos, S. K., Karpen, J. T., DeLuca, E. E., Golub, L., \& Hamilton, P. 2003, ApJ, 590, 547

Brown, J. C. 1971, SoPh, 18, 489

Carlsson, M., \& Stein, R. F. 1997, ApJ, 481, 500

Cheung, M. C. M., Boerner, P., Schrijver, C. J., et al. 2015, ApJ, 807, 143

De Pontieu, B., Erdélyi, R., \& de Wijn, A. G. 2003, ApJL, 595, L63

Del Zanna, G. 2013, A\&A, 558, A73

Falchi, A., Qiu, J., \& Cauzzi, G. 1997, A\&A, 328, 371

Fletcher, L., \& de Pontieu, B. 1999, ApJL, 520, L135

Fletcher, L., \& Hudson, H. S. 2008, ApJ, 675, 1645

Hannah, I. G., Hudson, H. S., Battaglia, M., et al. 2011, SSRv, 159, 263

Krucker, S., Hudson, H. S., Jeffrey, N. L. S., et al. 2011, ApJ, 739, 96

Lemen, J. R., Title, A. M., Akin, D. J., et al. 2012, SoPh, 275, 17

Neupert, W. M. 1968, ApJL, 153, L59

Pesnell, W. D., Thompson, B. J., \& Chamberlin, P. C. 2012, SoPh, 275, 3

Polito, V., Testa, P., Allred, J., et al. 2018, ApJ, 856, 178

Reale, F., Testa, P., Petralia, A., \& Graham, D. R. 2019, ApJ, in press (arXiv:1907.02291)

Reep, J. W., Polito, V., Warren, H. P., \& Crump, N. A. 2018, ApJ, 856, 149

Scherrer, P. H., Schou, J., Bush, R. I., et al. 2012, SoPh, 275, 207

Testa, P., De Pontieu, B., Allred, J., et al. 2014, Sci, 346, B315

Testa, P., De Pontieu, B., Martínez-Sykora, J., et al. 2013, ApJL, 770, L1

Tripathi, D., Mason, H. E., Young, P. R., Chifor, C., \& Del Zanna, G. 2008a, in ASP Conf. Ser. 397, First Results From Hinode, ed. S. A. Matthews, J. M. Davis, \& L. K. Harra (San Francisco, CA: ASP), 45

Tripathi, D., Mason, H. E., Young, P. R., \& Del Zanna, G. 2008b, A\&A, 481, L53

Ugarte-Urra, I., \& Warren, H. P. 2014, ApJ, 783, 12

Uitenbroek, H. 2001, ApJ, 557, 389

Viall, N. M., \& Klimchuk, J. A. 2016, ApJ, 828, 76

Wright, P. J., Hannah, I. G., Grefenstette, B. W., et al. 2017, ApJ, 844, 132 